\section{Adenoviral melanoma differentiation- associated gene 7 induces apoptosis in lung cancer cells through mitochondrial permeability transition-independent cytochrome c release}

\author{
Abujiang Pataer, MD, $\mathrm{PhD}^{\mathrm{a}}$ \\ Sunil Chada, $\mathrm{PhD}^{\mathrm{c}}$ \\ Kelly K. Hunt, $M D^{b}$ \\ Jack A. Roth, MD ${ }^{a}$ \\ Stephen G. Swisher, MD ${ }^{\mathrm{a}}$
}

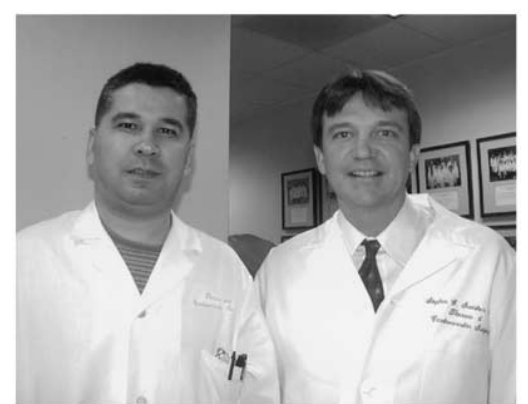

Drs Pataer and Swisher (left to right)
From the Section of Thoracic Molecular Oncology, Departments of Thoracic and Cardiovascular Surgery ${ }^{\mathrm{a}}$ and Surgical On-

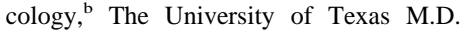
Anderson Cancer Center, and Introgen Therapeutics Inc, ${ }^{\mathrm{c}}$ Houston, Tex.

Supported by grants from the National Cancer Institute and the National Institutes of Health (P01 CA78778-01A1 to J.A.R. and S.G.S., SBIR S/C 1R43 CA86587-1 to S.G.S. and S.C., and SPORE 2P50CA70970-04), by gifts to the Division of Surgery from Tenneco and Exxon for the Core Laboratory Facility, by the University of Texas M. D. Anderson Cancer Center Support Core Grant (CA 16672), by donations from the Charles Rogers Memorial and Gene Therapy Donor funds, by a grant from the Tobacco Settlement Funds as appropriated by the Texas State Legislature (Project 8), by the W. M. Keck Foundation, and by a sponsored research agreement with Introgen Therapeutics, Inc (SR93-0041).

Read at the Eighty-second Annual Meeting of The American Association for Thoracic Surgery, Washington, DC, May 5-8, 2002.

Received for publication May 20, 2002; revisions requested July 8, 2002; revisions received July 22, 2002; accepted for publication Aug 14, 2002.

Address for reprints: Stephen G. Swisher, MD, Department of Thoracic and Cardiovascular Surgery, The University of Texas M.D. Anderson Cancer Center, 1515 Holcombe Blvd, Box 445, Houston, TX 77030 (E-mail: sswisher@mdanderson.org).

J Thorac Cardiovasc Surg 2003;125: 1328-35

Copyright $\odot 2003$ by The American Association for Thoracic Surgery

$0022-5223 / 2003 \$ 30.00+0$

doi:10.1016/S0022-5223(02)73247-9
Objective: Melanoma differentiation-associated gene 7 is a novel tumor suppressor gene that induces apoptosis in lung cancer cells when delivered by adenoviral gene transfer as Ad-mda7. The mechanisms of action are not well defined but may involve release of cytochrome $\mathrm{c}$ from the mitochondria with subsequent caspase activation.

Methods: The lung cancer cell lines A549 and H1299 were transduced with Ad-mda7, adenovirus containing the gene for p53 (Ad-p53), and control adenoviral luciferase vectors. Staurosporine was used as a positive control to induce cytochrome $\mathrm{c}$ release through mitochondrial permeability transition-dependent pores, whereas cyclosporine (INN: ciclosporin) was used to specifically inhibit these mitochondrial permeability transition-dependent pores. Apoptosis was evaluated with fluorescence-activated cell sorting analysis of subdiploid populations and mitochondrial membrane potential changes with tetramethylrhodamine ethylester perchlorate.

Results: Melanoma differentiation-associated gene 7, transduced by Ad-mda7 into H1299 and A549 lung cancer cells, resulted in sharp increases in cytosolic cytochrome c levels followed by induction of apoptosis and cellular death. The release of cytochrome $\mathrm{c}$ from the mitochondria occurred without changes in the mitochondrial membrane potential. Unlike staurosporine treatment, transduction with Ad-p53 and Ad-mda7 caused releases of cytochrome $\mathrm{c}$ and apoptosis that were not blocked by cyclosporine, suggesting a mitochondrial permeability transition pore-independent pathway.

Conclusions: Ad-mda7 induces apoptosis in lung cancer cells through mitochondrial cytochrome $\mathrm{c}$ release in a process that is not dependent on mitochondrial membrane potential changes and occurs through mitochondrial permeability transition-independent pores. This unique mechanism of action may allow treatment of patients with lung cancer resistant to mitochondrial permeability transition-dependent cell death processes.

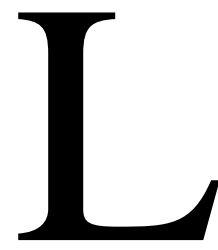

ocoregionally advanced lung cancer remains a significant clinical problem with few therapeutic options. Conventional therapy with surgery, chemotherapy, or radiotherapy has been ineffective in obtaining a cure for most patients. ${ }^{1,2}$ The advent of gene therapy offers a novel mode of therapy to treat lung cancer through the transfer of functional genes. Phase I and II clinical trials in lung cancer have demonstrated the feasibility of transferring wild-type p53 with adenoviral vectors 
(Ad-p53) to induce apoptosis and cellular death. ${ }^{3-5}$ Unfortunately, some patients are resistant to Ad-p53 gene transfer because of molecular changes in downstream mediators of apoptosis. Thus there is a need for other genes to be identified that can treat patients with lung cancer resistant to p53 gene transfer. Melanoma differentiation-associated gene 7 (mda-7) is a novel tumor suppressor gene that was first identified by subtraction hybridization of a melanoma cell line treated with interferon $\beta$ and mezerin. ${ }^{6}$ Adenoviral transfer of mda-7 (Ad-mda7) induces apoptosis in a wide range of cancer cells, including p53-sensitive (H1299) and p53-resistant (A549) lung cancer cells. ${ }^{7}$ The mechanism by which Ad-mda7 induces apoptosis, however, is not well defined.

Because the mitochondrial release of cytochrome $\mathrm{c}$ is a critical step in the cell's commitment to apoptosis and cellular death, we focused on the effects of Ad-mda7 on the mitochondria and compared them with those of other known apoptotic inducers. One class of proapoptotic stimuli (such as $\mathrm{p} 53, \mathrm{Bax}, \mathrm{Bak}$, and staurosporine) are dependent on mitochondrial membrane potential (MMP) changes to induce opening of mitochondrial permeability transition (MPT)-dependent pores, which then release cytochrome $\mathrm{c}$ and other apoptogenic factors. ${ }^{8-10}$ MPT-dependent pores are multiprotein complexes that span the inner and outer mitochondrial membranes and allow the release of cytochrome $\mathrm{c}$ into the cytosol from the intramitochondrial space after MMP changes. In this class of apoptosis-inducing agents, the release of cytochrome $\mathrm{c}$ through MPT-dependent pores can be blocked by cyclosporine (INN: ciclosporin) and bongkrekic acid, inhibiting the onset of apoptosis and cellular death. Other apoptosis-inducing agents, such as Bid, however, may not be dependent on MMP changes to induce cytochrome c release and appear to be able to release cytochrome $\mathrm{c}$ through MPT-independent pores that are not blocked by cyclosporine or bongkrekic acid .11,12

In this study we found that Ad-mda7 induced cytochrome $\mathrm{c}$ release through MPT-independent pores that were not blocked by cyclosporine. This unique mechanism of action occurred in both p53-sensitive (H1299) and p53resistant (A549) cell lines, which provides some insight into molecular differences of action between Ad-mda7 and Ad$\mathrm{p} 53$. The ability of Ad-mda7 to induce cytochrome c release through MPT-independent pores may allow a novel means of treating patients with lung cancer resistant to Ad-p53 and other MPT-dependent cell death processes.

\section{Material and Methods}

\section{Cell Lines and Immunoblot Assay}

A549 (p53 resistant) and H1299 (p53 sensitive) human lung cancer cell lines were obtained from the American Type Culture Collection (American Type Culture Collection, Manassas, Va). All cells were maintained in Roswell Park Memorial Institute medium 1640 supplemented with $10 \%$ fetal bovine serum, $10-\mathrm{mmol} / \mathrm{L}$ glu- tamine, $100-\mathrm{U} / \mathrm{mL}$ penicillin, and $100-\mu \mathrm{g} / \mathrm{mL}$ streptomycin (Life Technologies, Inc, Rockville, Md) in a 5\% carbon dioxide atmosphere at $37^{\circ} \mathrm{C}$.

Immunoblot assays were performed with total cell lysates, which were prepared by lysing cell monolayers in plates with sodium dodecylsulfate (SDS) polyacrylamide gel electrophoresis (PAGE). Each lane was loaded with $5 \mu \mathrm{g}$ of cell lysate protein, as determined by BCA protein assay (Pierce Chemical Company, Rockford, Ill). Subsequently, proteins were separated under reducing conditions by SDS-PAGE. After electrophoresis at $20 \mathrm{~mA}$ for 2 hours, the proteins in the gels were transferred to Hybond-ECL membranes (Amersham Pharmacia Biotech, Inc, Piscataway, NJ). Then the membranes were blocked with $1 \%$ dry milk and $0.1 \%$ polysorbate 20 (Sigma, St Louis, Mo) and incubated with primary antibodies. The membranes were then incubated with secondary antibody conjugated with horseradish peroxidase (Amersham). The membranes were developed according to the Amersham ECL protocol. Relative quantities of protein were determined with a densitometer (Molecular Dynamics, Inc, Sunnyvale, Calif). Antibodies to the following were used: Bax, Bak, Bcl-2, Fas, FasL, and $\beta$-actin (Santa Cruz Biotechnology, Inc, Santa Cruz, Calif); cytochrome c (BD PharMingen, San Diego, Calif); and FADD, tumor necrosis factor (TNF) receptor 1 , TNF- $\alpha$, and TRADD (BioSource International, Inc, Camarillo, Calif).

\section{Adenovirus Production and Transduction}

Construction methods of the adenovirus vectors Ad-mda7, Adp53, Ad-LacZ, and Ad-luc (luciferase) have been reported previously elsewhere. ${ }^{13}$ The transduction efficiencies of the cell lines were determined with Ad-LacZ. Subsequent experiments used viral titers needed to transduce at least $70 \%$ of the cells.

\section{Apoptosis and Cellular Viability Assays}

We measured apoptotic cells by propidium iodide staining and fluorescence-activated cell sorting (FACS) analysis. Cells were harvested, reduced to pellets by centrifugation, and resuspended in phosphate-buffered saline solution (PBS) containing $50-\mu \mathrm{g} / \mathrm{mL}$ propidium iodide, $0.1 \%$ Triton X-100 (The Dow Chemical Company, Midland, Mich), and $0.1 \%$ sodium citrate. Samples were stored at $4{ }^{\circ} \mathrm{C}$ for 16 hours and vortex mixed before FACS analysis (FACScan, FL-3 channel; BD Immunocytometry Systems, San Jose, Calif). Cells were grown in 96-well plates in $100 \mu \mathrm{L}$ volume/ well. On day 1, cells were transduced with Ad-mda7, Ad-p53, or control vectors. On day 2, the cells were incubated with the tetrazolium salt XTT for 4 to 24 hours according to the Roche protocol (Roche Diagnostics GmbH, Mannheim, Germany). Viability was assessed spectrophotometrically on an enzyme-linked immunosorbent assay plate reader.

\section{MMP Measurements}

The potential-sensitive fluorochrome tetramethylrhodamine ethylester perchlorate (TMRE; Molecular Probes, Inc, Eugene, Ore) was used to measure MMP changes. Cells were incubated with 25-nmol/L TMRE for 30 minutes at $37^{\circ} \mathrm{C}$ in the dark, washed in PBS, and analyzed by FACS on the FL- 2 channel (FACScan). ${ }^{14}$

\section{Cytochrome c Release Measurements}

Release of cytochrome c from mitochondria was measured by immunoblotting. Cells were harvested by centrifugation and gently 


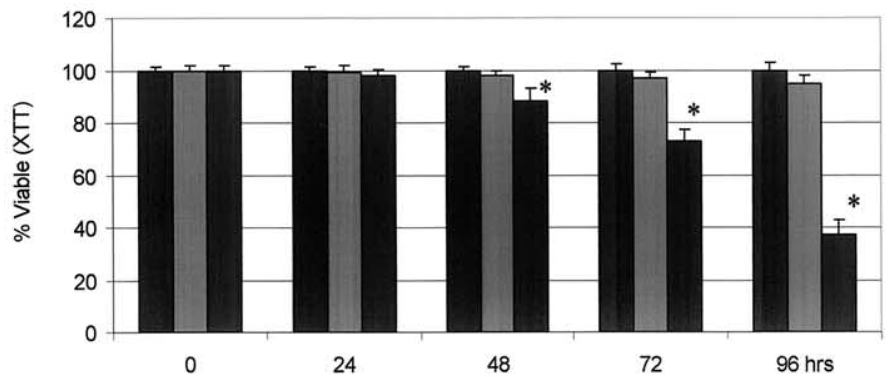

A

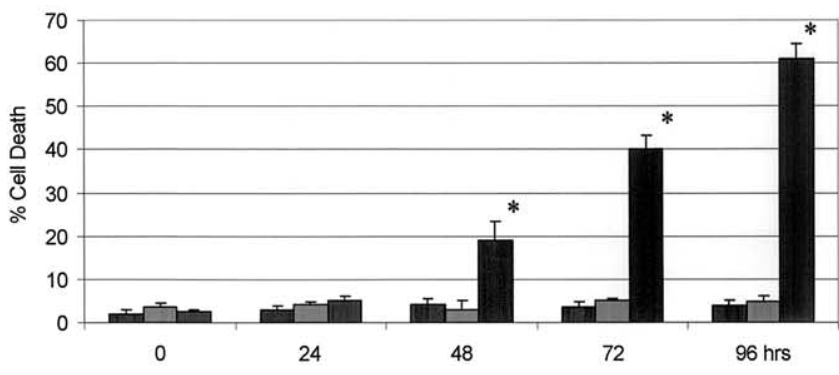

B

-PBS $\square$ Ad-luc $\square$ Ad-mda7

Figure 1. Adenovirally mediated overexpression of mda-7 suppresses proliferation and induces cell death in lung cancer cells. A, Cell viability was determined with XTT assay in A549 cells after treatment with PBS, Ad-luc (2500 vp), or Ad-mda7 (2500 vp). B, Percentage of cell death. Cells were analyzed by flow cytometry after transduction. Triplicate experiments were performed for each cell line. Bar heights represent mean; error bars represent SD. Asterisk indicates $\boldsymbol{P}<.05$.

A
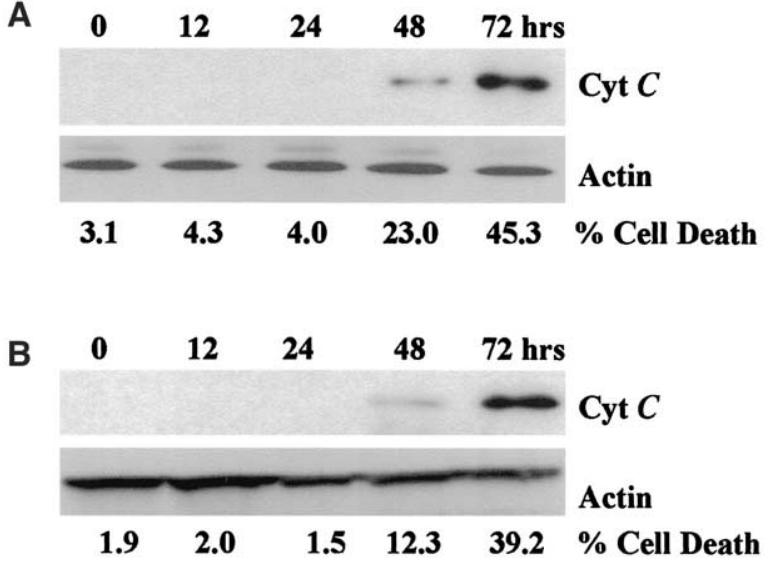

Figure 2. Kinetics of cytochrome c (Cyt $C$ ) release induced by Ad-mda7. Cytosolic extracts from A549 (A) and H1299 (B) cells were prepared for analysis of cytochrome c release, and DNA fragmentation was also assessed by propidium staining and flow cytometry.

lysed for 5 minutes in an ice-cold buffer containing $25-\mathrm{mmol} / \mathrm{L}$ tris(hydroxymethyl)aminomethane and $5-\mathrm{mmol} / \mathrm{L}$ magnesium chloride, $\mathrm{pH}$ 7.4. Lysates were centrifuged for 5 minutes at $16,000 \mathrm{~g}$, supernatants were mixed with $1 \times$ Laemmli reducing SDS-PAGE sample buffer, and extracts from equal numbers of cells $\left(10-20 \times 10^{6}\right)$ were resolved by $15 \%$ SDS-PAGE. Polypeptides were transferred to nitrocellulose membranes $(0.2 \mu \mathrm{mol} / \mathrm{L}$; Schleicher \& Schuell Inc, Keene, NH), and cytochrome c was detected by immunoblotting with the monoclonal antibody clone 7H8.2C12 (BD PharMingen).

\section{Statistical Analysis}

The data reported in the figures represent the means of three independent experiments with SDs. Analysis of variance and the two-tailed Student $t$ test were used for statistical analysis of multiple groups and pairwise comparisons, respectively.

\section{Results}

Ad-mda7 Induction of Apoptosis and Cytochrome c Release from Mitochondria

Cellular viability was assessed with the XTT assay. Transduction of both the p53-resistant (A549) and p53-sensitive (H1299, data not shown) lung cancer cell lines resulted in marked decrease in cellular viability 48 to 72 hours after infection (Figure 1, A). Induction of apoptosis induction was evaluated by flow cytometric analysis of subdiploid populations. Both A549 and H1299 (data not shown) lung cancer cells demonstrated apoptosis induction 48 to 96 hours after Ad-mda7 transduction. No apoptosis was seen in Ad-luctreated or PBS-treated cells (Figure 1,B). Transduction of both A549 and H1299 lung cancer cell lines with Ad-mda7 resulted in cytochrome $\mathrm{c}$ release into the cytosol 48 hours after treatment. This corresponded to the onset of apoptosis and demonstrated involvement of the mitochondria in the apoptotic cascade (Figure 2). Only Ad-mda7-transduced cells demonstrated mda-7 protein expression by Western blotting (data not shown).

\section{Ad-mda7's Effects on MMP Changes and Apoptosis}

Disruption of the MMP is an essential event in the development of apoptosis after treatment with MPT-dependent apoptotic agents. Staurosporine was used as a positive control for MPT-dependent apoptotic agents. In H1299 cells, both Ad-p53 and staurosporine induced MMP changes and apoptosis, presumably through release of cytochrome $\mathrm{c}$ through MPT-dependent pores. Both the MMP changes and apoptosis were inhibited by cyclosporine, which selectively blocks MPT-dependent pores (Figures 3, A, and 4, A). Ad-mda7, however, did not induce MMP changes but was still able to induce apoptosis that was not inhibited by cyclosporine. In A549 cells, Ad-p53 was unable to induce 


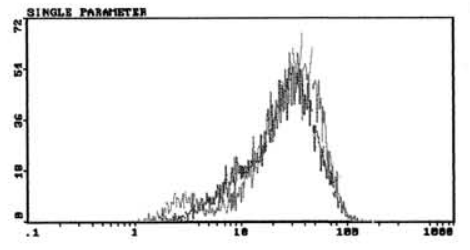

\section{Ad-mda7}

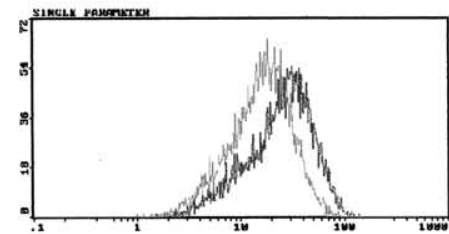

Adp53

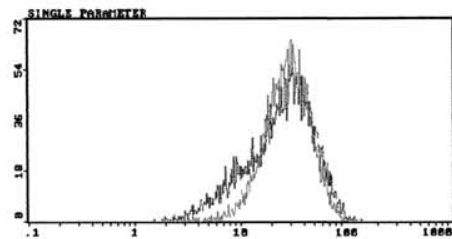

A

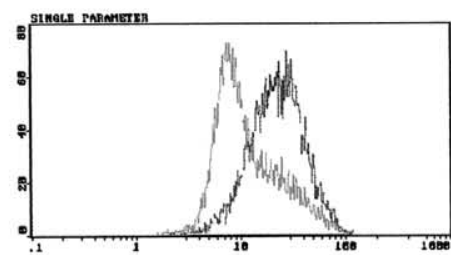

Adp53 + CsA

Ad-mda7

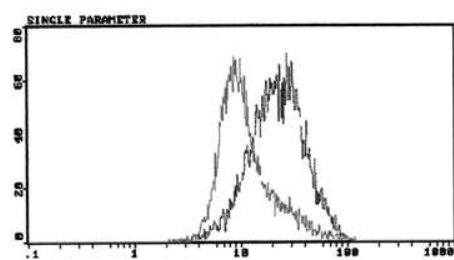

Ad-mda7 + CsA
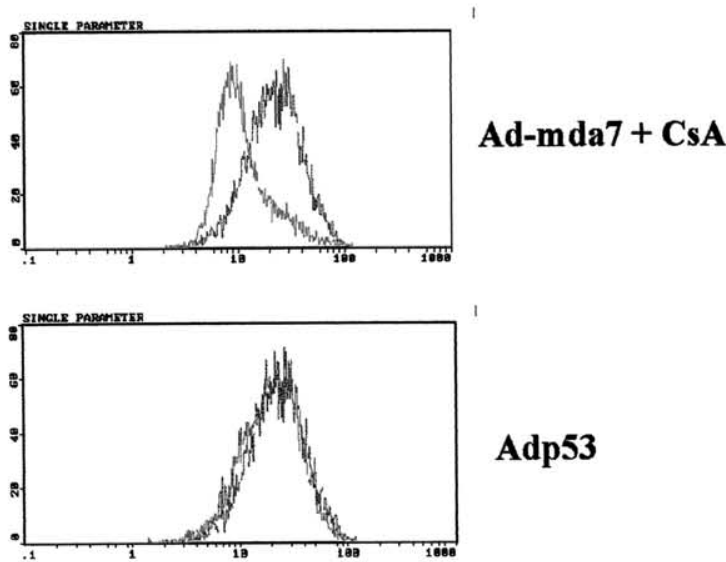

Adp53

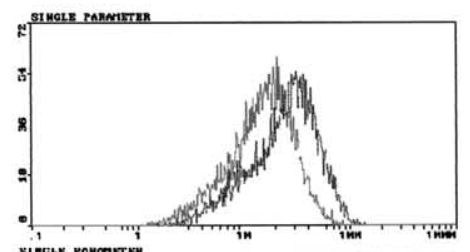

ST

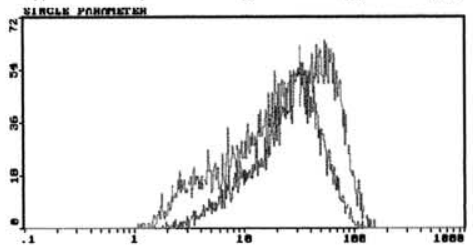

ST+CsA

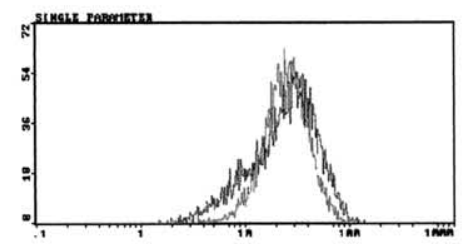

CsA

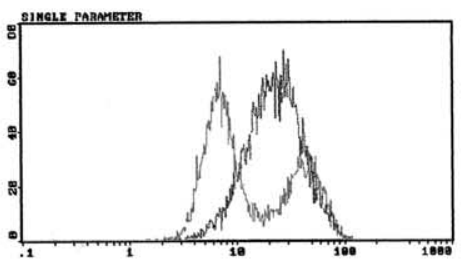

ST

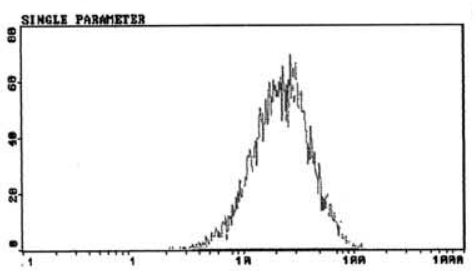

ST+CsA

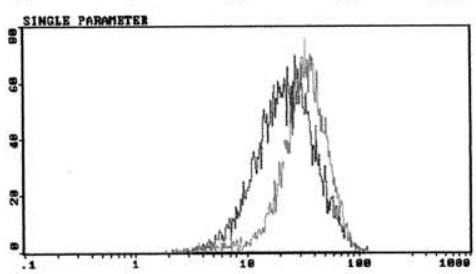

CsA

Figure 3. Effect of Ad-mda7 on MMP. Measurement of MMP after treatment with Ad-mda7, Ad-p53, and staurosporine (ST, $1 \mu \mathrm{mol} / \mathrm{L}$ ). Where indicated, cells were pretreated with cyclosporine (CsA, $10 \mu \mathrm{mol} / \mathrm{L})$. H1299 (A) and A549 (B) cells were stained with potential-sensitive dye TMRE, and fluorescence was assessed by flow cytometry. Triplicate experiments were performed for each cell line. Bar heights represent mean; error bars represent SD.

apoptosis or MPT changes (Figures 3, $B$, and 4, B). We used staurosporine as a positive control because it induces MMP changes and apoptosis in H1299 and A549 cells, both of which are blocked by cyclosporine, presumably because of inhibition of MPT-dependent pores. Interestingly, Ad-mda7 does induce MMP changes, but these changes are not re- versed by cyclosporine. Additionally, cyclosporine is unable to inhibit Ad-mda7-induced apoptosis. Taken together, these data suggest that Ad-mda7-induced apoptosis and cytochrome c release may occur through MPT-independent pores that are not blocked by cyclosporine. 

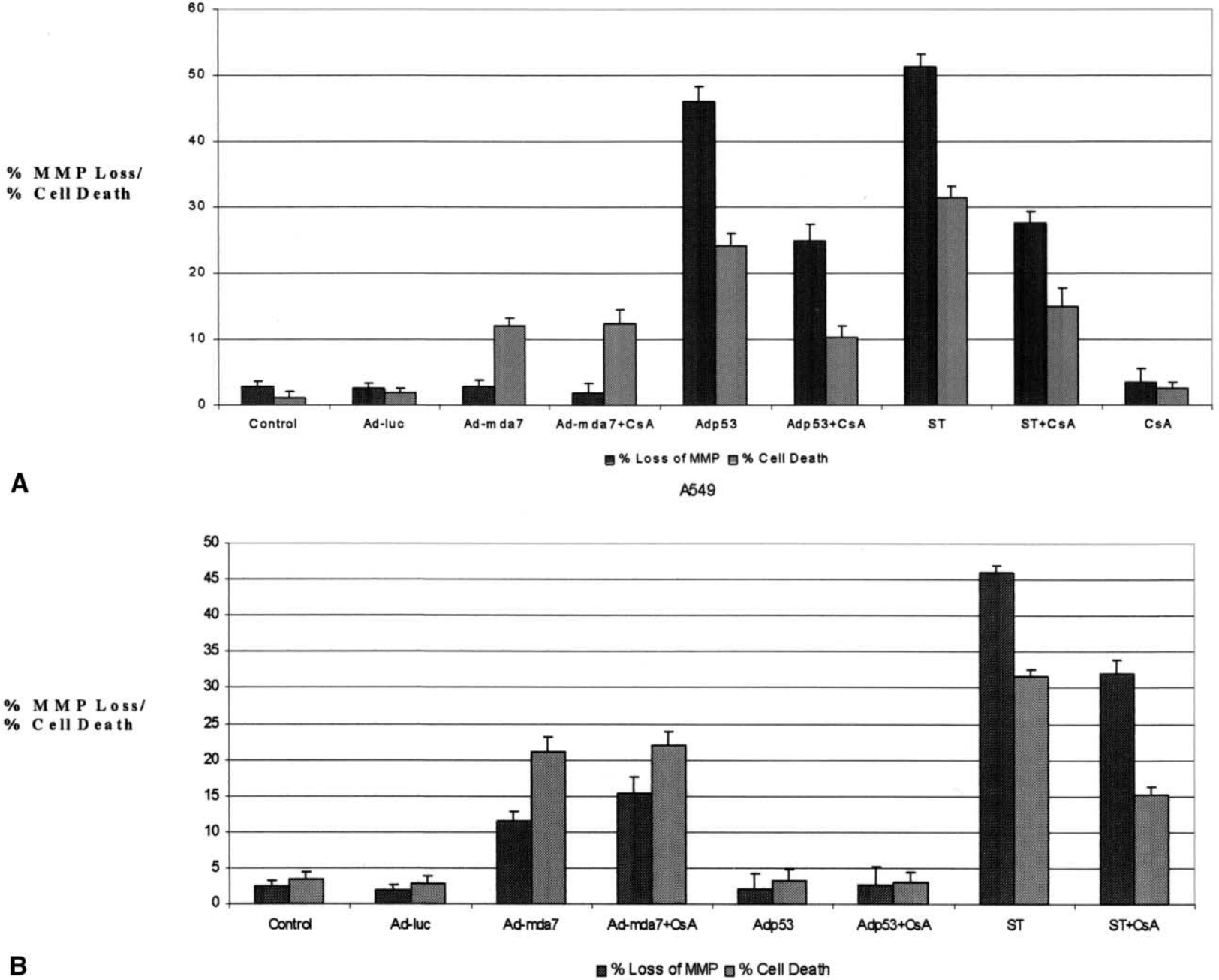

Figure 4. Cyclosporine (CsA) does not prevent loss of MMP. H1299 (A) and A549 (B) cells were treated with Ad-mda7, Ad-p53, and staurosporine (ST, $1 \mu \mathrm{mol} / \mathrm{L}$ ). Where indicated, cells were pretreated with cyclosporine (CsA, $10 \mu \mathrm{mol} / \mathrm{L}$ ). After times indicated, cells were lysed, and MPP was determined with fluorescent dye TMRE as described in Material and Methods section.

\section{Ad-mda7 Upward Regulation of Extrinsic Pathway in A549 Cells}

To determine whether Ad-mda7 activated the mitochondria through upward regulation of the Bcl-2 family of genes or the death receptor pathway in A549 cells, Ad-mda7-treated cells (A549) were assessed by immunoblot assay for changes in Bak, Bax, Bcl-2, TNF- $\alpha$, TNF receptor 1, TRADD, FasL, Fas, and FADD expressions (Figure 5). No differences in Bcl-2 family members were seen in A549 cells other than Bid activation, as previously reported, ${ }^{6}$ and significant upward regulation of FasL (Figure 5). Although Fas receptor function was not evaluated in A549 cells, we did observe caspase 8 activation and apoptosis inhibition by caspase 8 inhibitors (data not shown), which suggests up- ward regulation of the extrinsic death receptor pathway in A549 cells after Ad-mda7 transduction.

\section{Discussion}

Gene therapy offers the opportunity of a new modality of therapy for locoregionally advanced lung cancer. In a recently completed phase II trial that combined Ad-p53 with radiation therapy, more than $60 \%$ of the tumors demonstrated no viable tumor in core biopsy specimens 3 months after treatment. ${ }^{4,5}$ It is clear, however, that not all patients have a response to Ad-p53, and some with an initial response to Ad-p53 ultimately have disease progression despite continued treatment. Additionally, preclinical experience with lung cancer cell lines has demonstrated p53 


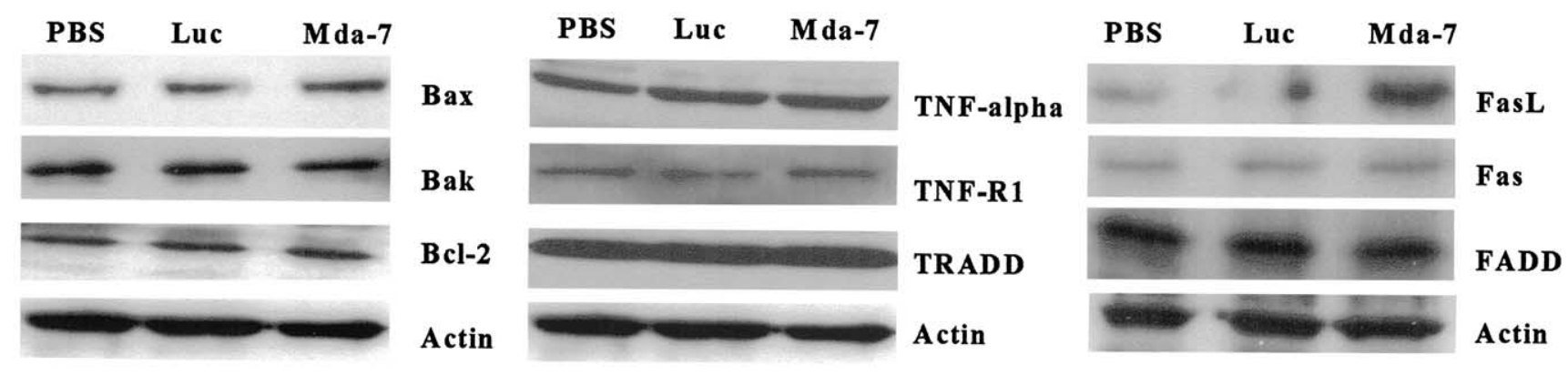

Figure 5. Ad-mda7 induces FasL. A, Western blot analysis of expressions of Bax, Bak, Bcl-2, TNF- $\alpha$, TNF receptor 1 (TNF-R1), TRADD, FasL, Fas, and FADD in A549 cell lysates 48 hours after treatment with PBS, Ad-luc, or Ad-mda7. Expression level of actin was used as control.

resistance in a number of cell lines, including the A549 human lung cancer cell line. ${ }^{15}$ The reasons for this resistance have not been delineated. We therefore evaluated the effectiveness of adenoviral transfer of the novel tumor suppressor gene mda-7 and sought to determine the molecular mechanisms of action differentiating this gene from other classic apoptosis-inducing agents.

In this study we chose to focus on the mitochondria, because many investigators have suggested that mitochondrial activation and cytochrome c release are the critical steps in cellular commitment to apoptosis. ${ }^{16-18}$ Several proapoptotic genes, including Bax, Bak, and p53, are believed to induce MMP changes that open MPT-dependent pores that allow the release of cytochrome $\mathrm{c}$ and formation of an apoptosome with cytochrome c, APAF-1, and caspase 9 (Figure 6). ${ }^{8-10}$ This apoptosome activates caspases 3, 6, and 7 , leading to the executioner phase of apoptosis with cleavage of a variety of cellular substrates, including PARP, DFF 45, and gelsolin. These classic proapoptotic agents can be inhibited by cyclosporine or bongkrekic acid, which block the MPT-dependent pores, preventing MMP changes and cytochrome c release. ${ }^{9,10}$ Previous studies have demonstrated that cytochrome $\mathrm{c}$ is not released after Ad-p53 treatment in resistant A549 cells, suggesting a possible defect at the mitochondrial level of action. ${ }^{15}$ Our finding of cytochrome c release after Ad-mda7 treatment is interesting because it suggests that Ad-mda7 is able to induce apoptosis through cytochrome c release despite Ad-p53 resistance. This ability to induce killing in p53-resistant cells has potential therapeutic implications, because Ad-mda7 appears to act through a unique mechanism.

Our findings suggest that Ad-mda7 induces cytochrome c release from the mitochondria into the cytosol through MPT-independent pores, because cyclosporine is unable to inhibit apoptosis or cytochrome c release after Ad-mda7 transduction (Figure 4, $A$ and $B$ ) but is fully capable of blocking the classic MPT-dependent apoptosis agents staurosporine and Ad-p53. How Ad-mda7 induces apoptosis through MPT-independent pores is not clear, but the induc- tion may be due in part to activation of the extrinsic death receptor pathway. It is important to remember that this may be cell-line specific (A549 cells), because other authors have demonstrated Ad-mda7-induced apoptosis in other cell lines in the absence of Bid cleavage. ${ }^{13}$ In A549 cells we saw no changes in levels of Bax or Bak, which are known to act by releasing cytochrome $\mathrm{c}$ through MPT-dependent pores inhibitable by cyclosporine or bongkrekic acid. ${ }^{10}$ We noted, however, increases in FasL, which can act through the extrinsic death receptor pathway by activating caspase 8 , leading to Bid cleavage and mitochondrial activation (Figure 6). This is consistent with our previously reported observation of caspase 8 activation and Bid cleavage after Ad-mda7 transduction of A549 lung cancer cells, mediated in part through upward regulation of the interferon-inducible double-stranded RNA protein kinase and other protein intermediaries, although in a longer time course (48 to 96 hours) than such direct apoptosis agents such as Ad-Bak. ${ }^{6}$ Several authors have observed that Bid, unlike Bak or Bax, induces cytochrome $\mathrm{c}$ release through a pathway independent of MPT-dependent pores ${ }^{11,12}$ perhaps by oligomerizing Bak or Bax into their own MPT-independent pores in the outer mitochondrial membrane. ${ }^{19}$

We have previously reported that adenovirally mediated overexpression of Bak or Bax could induce apoptosis in p53-sensitive and p53-resistant lung cancer cells, suggesting that other MPT-dependent apoptotic agents are able to induce a killing effect in A549 cells. ${ }^{15,20}$ These observations suggest that the defect in Ad-p53 killing of A549 cells may be above the mitochondrial checkpoint, because staurosporine, Ad-Bax, and Ad-Bak induce mitochondrial activation and apoptosis, presumably through functional MPT-dependent pathways. From a therapeutic standpoint Ad-mda7 has a significant advantage over Ad-Bak or Ad-Bax, because both of the latter vectors also induce killing of normal cells, whereas Ad-mda7 spares normal cells. Ad-mda7 is therefore able to kill p53-resistant A549 cells in a tumor-selective fashion, without the toxicity associated with Ad-Bax or Ad-Bak. 


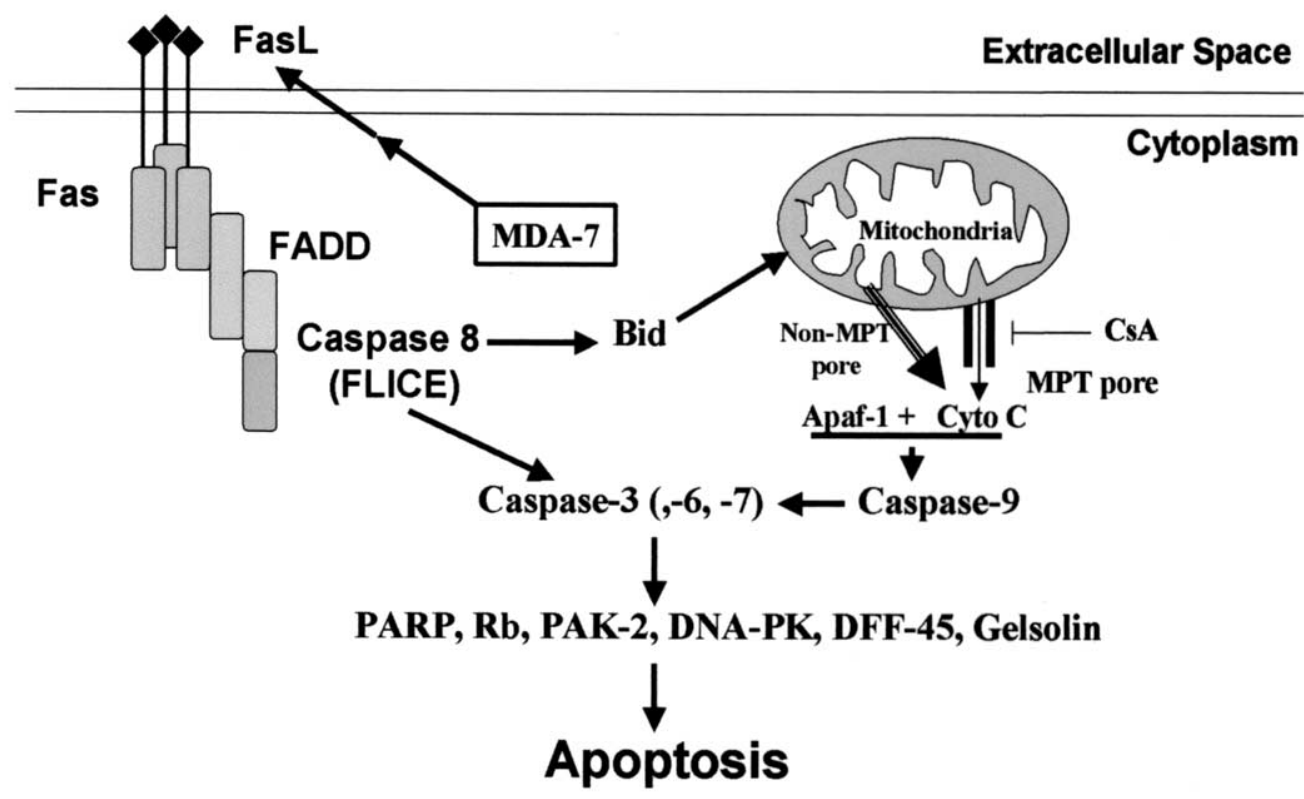

Figure 6. Diagram of postulated mitochondrial mechanism of action of Ad-mda7 in A549 cells with induction of FasL, caspase 8, Bid, and cytochrome c (Cyto C) release through MPT-independent pores not inhibitable by cyclosporine (CsA). Release of cytochrome c allows formation of apoptosome with Apaf-1, caspase 9, and cytochrome c leading to subsequent caspase 3, 6, and 7 activation and apoptosis induction.

In summary, we have shown that adenovirally mediated overexpression of mda-7 leads to rapid induction of apoptosis in p53-resistant and p53-sensitive lung cancer cells. Ad-mda7's mitochondrial mechanism appears to act through MPT-independent pore release of cytochrome c, with subsequent activation of executioner caspases and cellular death. The upward regulation of FasL, caspase 8 activation and Bid cleavage, suggests that Ad-mda7 may be acting preferentially through the extrinsic death receptor pathway in A549 cells, with subsequent mitochondrial activation and MPT-independent cytochrome $\mathrm{c}$ release. This unique mechanism of action may provide a novel means of treating patients with lung cancer resistant to p53 and other MPT-dependent cell death processes.

We thank Debbie Smith for her assistance in the preparation of the manuscript.

\section{References}

1. Le Chevalier T, Arriagada R, Quoix E, Ruffie P, Martin M, Tarayre $\mathrm{M}$, et al. Radiotherapy alone versus combined chemotherapy and radiotherapy in nonresectable non-small-cell lung cancer: first analysis of a randomized trial in 353 patients. J Natl Cancer Inst. 1991;83:417-423.

2. Roth JA, Fossella F, Komaki R, Ryan MB, Putnam JB, Lee JS, et al. A randomized trial comparing perioperative chemotherapy and surgery with surgery alone in resectable stage IIIA non-small-cell lung cancer. J Natl Cancer Inst. 1994;86:673-80.

3. Swisher SG, Roth JA, Nemunaitis J, Lawrence DD, Kemp BL, Carrasco $\mathrm{CH}$, et al. Adenovirus-mediated p53 gene transfer in ad- vanced non-small-cell lung cancer. J Natl Cancer Inst. 1999;91:76371.

4. Swisher SG, Roth JA, Komaki R, et al. A phase II trial of adenoviral mediated p53 gene transfer (RPR/INGN 201) in conjunction with radiation therapy in patients with localized non-small-cell lung cancer (NSCLC). Proc Am Soc Clin Oncol. 2000;19:461a.

5. Swisher SG, Roth JA, Komaki R, et al. Induction of pro-apoptotic mediators and tumor regression following intratumoral delivery of adenoviral p53 (RPR/INGN 201) and radiation therapy in patients with non-small-cell lung cancer (NSCLC). Proc Am Soc Clin Oncol. 2000;20:257a.

6. Jiang H, Lin JJ, Su ZA, Goldstein NI, Fisher PB. Subtraction hybridization identifies a novel melanoma differentiation associated gene, mda-7, modulated during human melanoma differentiation, growth and progression. Oncogene. 1995;11:2477-86.

7. Pataer A, Vorburger SA, Barber GN, Chada S, Mhashilkar AM, Zou-Yang $\mathrm{H}$, et al. Adenoviral transfer of the melanoma differentiation-associated gene 7 ( $m d a 7$ ) induces apoptosis of lung cancer cells via up-regulation of the double-stranded RNA dependent protein kinase (PKR). Cancer Res. 2002;62:2239-43.

8. Henry H, Thomas A, Shen Y, White E. Regulation of the mitochondrial checkpoint in $\mathrm{p} 53$-mediated apoptosis confers resistance to cell death. Oncogene. 2002;21:748-60.

9. Sugrue MM, Wang Y, Rideout HJ, Chalmers-Redman RM, Tatton WG. Reduced mitochondrial membrane potential and altered responsiveness of a mitochondrial membrane megachannel in p53-induced senescence. Biochem Biophys Res Comm. 1999;261:123-30.

10. Tsujimoto Y, Shimizu S. VDAC regulation by the Bcl-2 family of proteins. Cell Death Diff. 2000;7:1174-81.

11. Proapoptotic BH3-only Bcl-2 family members induce cytochrome c release, but not mitochondrial membrane potential loss, and do not directly modulate voltage-dependent anion channel activity. Proc Natl Acad Sci U S A. 2000;97:577-82.

12. Kim TH, Zhao Y, Barbers MJ, Kuharsky DK, Yin XM. Bid-induced cytochrome c release is mediated by a pathway independent of mito- 
chondrial permeability transition pore and Bax. J Biol Chem. 2000; 275:39474-81.

13. Mhashilkar AM, Schrock RD, Hindi M, Liao J, Sieger K, Kourouma $\mathrm{F}$, et al. Melanoma differentiation associated gene (mda7): a novel anti-tumor gene for cancer gene therapy. Mol Med. 2001;7:271-82.

14. Scadulto RC Jr, Grotyohann LW. Measurement of mitochondrial membrane potential using fluorescent rhodamine derivatives. Biophys J. 1999;76:469-77.

15. Kagawa S, Gu J, Swisher SG, Ji L, Roth JA, Lai D, et al. Antitumor effect of adenovirus-mediated Bax gene transfer on $p 53$-sensitive and p53-resistant cancer lines. Cancer Res. 2000;60:1157-61.

16. Marchetti P, Castedo M, Susin SA, Zamzami N, Hirsch T, Macho A, et al. Mitochondrial permeability transition is a central coordinating event of apoptosis. J Exp Med. 1996;184:1155-60.

17. Kroemer G, Reed JC. Mitochondrial control of cell death. Nat Med. 2000;6:513-9.

18. Desagher S, Martinou JC. Mitochondria as the central control point of apoptosis. Cell Biol. 2000;10:369-77.

19. Korsmeyer SJ, Wei MC, Saito M, Weiler S, Oh KJ, Schlesinger PH. Pro-apoptotic cascade activates BID, which oligomerizes Bak or Bax into pores that result in the release of cytochrome c. Cell Death Differ. 2000;7:1166-73.

20. Pataer A, Fang B, Yu R, Kagawa S, Hunt KK, McDonnell TJ, et al. Adenoviral Bak overexpression mediates caspase-dependent tumor killing. Cancer Res. 2000;60:788-92.

\section{Discussion}

Dr David R. Jones (Charlottesville, Va). I have a question regarding other strategies to more completely evaluate your proposed mechanism of action. Did you consider doing a cotransfection with a dominant-negative FADD or the treating the cells with CrmA to see whether this indeed would block the caspase 8 pathway? If so, did this subsequently affect your mitochondrial permeability?

Dr Swisher. That is a very interesting question, and to answer that, we are getting fibroblasts that are FADD deficient. We have used caspase 8 inhibitors and noted inhibition of caspase 8 activity and apoptosis in the A549 cells. It is important to keep in mind that upward regulation of the extrinsic death receptor pathway may be cell specific. Adenovirally transfected mda-7 can work in a variety of different ways in a variety of different cells. In the cell that I showed you, it went through the extrinsic death receptor pathway, but in other cells we can see upward regulation of Bak and Bcl-2. The common theme appears to be a little bit further down, at the mitochondrial level of action.

Dr Dao M. Nguyen (Bethesda, Md). These are intriguing findings, and I have a few questions. First, going back to the upward regulation of the Fas-Fas ligand pathway, have you tried to block it with blocking antibodies to see whether you can take away the apoptosis-inducing effect of this mda-7? The second question regards the time course of the study of the membrane depolarization. There are two models of mitochondrial membrane depolarization that are described and adopted in the literature. In one model, mitochondrial damage is mediated by opening of the voltage-dependent anion channels, leading to translocation of many apoptosis-inducing factors, including cytochrome c. In the other model, mitochondrial damage occurs after caspase activation. According to the second model, cytochrome c release occurs early, is not necessarily connected to mitochondrial depolarization, and is mediated by Bax/Bcl-2 in response to an apoptosis signal. Mitochondrial depolarization occurs only in the presence of complete caspase activation. I just wondered whether if you waited long enough beyond the 72-hour time point, you might start seeing the depolarization, which is a secondary phenomenon resulting from activation of caspases and not secondary to cytochrome $\mathrm{c}$ release.

Dr Swisher. As your question points out, this is a controversial area. I am not going to attempt to answer all the many different hypotheses that have been generated. The only thing that our data clearly showed was that cytochrome $\mathrm{c}$ was released from the mitochondria through pores that were not inhibitable by cyclosporine and were not always dependent on mitochondrial membrane changes. But there is, as you have mentioned, extensive discussion in the literature about mitochondrial membrane depolarization. Does everything have to go through those MMP-dependent pores? My own feeling is that there are probably multiple pores, both MMP dependent and independent.

With regard to the Fas ligand question, we have been able to induce apoptosis in these cells with Fas-inducing antibody. We have not tried yet with blocking antibodies, but that would be a good experiment.

Dr Peter K. Kim (Pittsburgh, Pa). I believe that A549 cells have a defect in expressing Fas on the surface, and I wonder whether your mda-7 is also upwardly regulating expression of Fas on the surface. Have you looked by immunohistochemical methods to see whether that is occurring?

Dr Swisher. At a Western blotting level, we saw upward regulation of Fas ligand, and it is purely speculative what is going on at the receptor level. But we do not have evidence, at least in Western blotting, of upward regulation of the actual Fas receptor.

Dr Kim. So there is Fas protein in the cells, but even though the total levels do not change, maybe you are getting more expression of Fas on the cell surface that is then interacting with the Fas ligand to kill the cells?

Dr Swisher. Right, and there is a variety of other potential things too. The mda-7 may be acting on the inside of the receptor, and it may have nothing to do with that upward regulation of Fas ligand. We do see caspase 8 activation in the A549 cells, and we do see Bid cleavage, which definitely means that the pathway is activated at least in A549 cells. There are reports in the literature that Bid can interact with Bax on the cell surface and allow cytochrome c release without MMP changes, so this may be the mechanism that is working with the A549 cells that we are evaluating.

Dr John H. Stewart (Bethesda, Md). You showed that Bid was cleaved; however, the total protein levels of Bax remained the same. Are you interested in the future at looking at conformational changes in Bax and to see whether your adenovirus is involved in that pathway of inducing cytochrome c release?

Dr Swisher. That is an interesting question, because cell lines differ in the response to Ad-mda7. We have looked at Ad-mda7 before in other cell lines, and we have seen upward regulation of Bax and Bak, but in certain cell lines mda-7 appears to preferentially work through the extrinsic death receptor. Whether that's cell-type specific we do not know for sure. But in this one cell line it did appear to be mostly through the extrinsic pathway and not through Bcl-2 and Bax and Bak pathway. 